\title{
A Smartphone App-Based Mindfulness Intervention for Cancer Survivors: Protocol for a Randomized Controlled Trial
}

Utkarsh B Subnis $^{1^{*}}, \mathrm{PhD}$, MBBS; Norman AS Farb ${ }^{2 *}, \mathrm{PhD}$; Katherine-Ann Laura Piedalue ${ }^{1^{*}}, \mathrm{BA}$; Michael Speca ${ }^{1^{*}}$, PsyD, C Psych; Sasha Lupichuk ${ }^{1 *}$, MD; Patricia A Tang ${ }^{1 *}$, MD; Peter Faris ${ }^{3^{*}}$, PhD; Mark Thoburn ${ }^{4 *}$, MJ; Bechara J $\mathrm{Saab}^{4 *}, \mathrm{PhD}$; Linda E Carlson ${ }^{1 *}, \mathrm{PhD}, \mathrm{RPsych}$

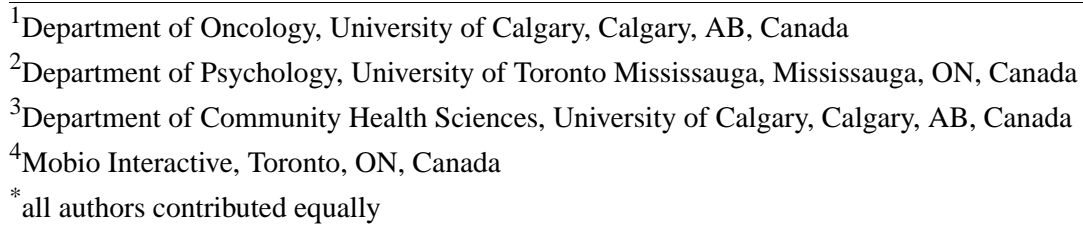

\section{Corresponding Author:}

Utkarsh B Subnis, PhD, MBBS

Department of Oncology

University of Calgary

22022 Street SW

Calgary, $\mathrm{AB}, \mathrm{T} 2 \mathrm{~S} 3 \mathrm{C} 1$

Canada

Phone: 14034762465

Email: utkarsh.subnis@ucalgary.ca

\section{Abstract}

Background: Cancer patients transitioning to survivorship after completing cancer treatments need psychosocial interventions to manage stressors such as anxiety, depression, and fear of cancer recurrence. Mindfulness-based interventions (MBIs) are effective for treating these symptoms; however, cancer survivors are often unable to participate in face-to-face interventions because of difficulties such as work and family commitments, treatment-related side-effects, scheduling conflicts, and geography. Smartphone app-based MBIs are an innovative way to deliver psychosocial cancer care and can overcome several such difficulties, since patients can participate at their own convenience.

Objective: The SEAMLESS (Smartphone App-Based Mindfulness Intervention for Cancer Survivors) study aims to evaluate the efficacy of a tailored app-based mindfulness intervention for cancer survivors (the Am Mindfulness-Based Cancer Survivorship-MBCS—Journey) for treating (1) symptoms of stress (primary outcome), as well as (2) fear of cancer recurrence, anxiety, depression, fatigue, and overall physical functioning (secondary outcomes). This is the first Canadian efficacy trial of a tailored mindfulness app intervention in cancer survivors.

Methods: This is a randomized waitlist-controlled trial, which will evaluate the effectiveness of Am MBCS for impacting the primary and secondary outcomes in cancer survivors who have completed all their cancer treatments. Outcomes will be assessed using web-based surveys with validated psychometric instruments at (1) baseline, (2) mid-intervention (2 weeks later), (3) immediately postintervention (4 weeks), (4) 3 months postbaseline, (5) 6 months postbaseline, and (6) 12 months postbaseline. The waitlist group will complete all assessments and will cross over to the intervention condition after the 3-month assessment. In addition, data will be obtained by the smartphone app itself, which includes users' engagement with the app-based intervention, their emotional state (eg, angry and elated) from a user-inputted digital emotion-mapping board, and psychobiometric data using photoplethysmography technology.

Results: The study received ethics approval in September 2018 and recruitment commenced in January 2019. Participants are being recruited through a provincial cancer registry, and the majority of participants currently enrolled are breast (44/83, 53\%) or colorectal $(17 / 83,20 \%)$ cancer survivors, although some survivors of other cancer are also present. Data collection for analysis of the primary outcome time-point will be complete by September 2019, and the follow-up data will be collected and analyzed by September 2020. Data will be analyzed to determine group differences using linear mixed modelling statistical techniques.

Conclusions: Cancer care providers are uncertain about the efficacy of app-based mindfulness interventions for patients, which are available in great supply in today's digital world. This study will provide rigorously evaluated efficacy data for an app-based 
mindfulness intervention for cancer survivors, which if helpful, could be made available for psychosocial care at cancer centers worldwide.

Trial Registration: ClinicalTrials.gov NCT03484000; https://clinicaltrials.gov/ct2/show/NCT03484000

International Registered Report Identifier (IRRID): DERR1-10.2196/15178

(JMIR Res Protoc 2020;9(5):e15178) doi: 10.2196/15178

\section{KEYWORDS}

mobile health; psycho-oncology; mindfulness; mind-body therapies

\section{Introduction}

\section{Background}

Previous research suggests that cancer survivors in Canada have several unmet psychosocial needs after completing treatments, which differ from patients newly diagnosed or undergoing treatment [1]. Almost half of all cancer survivors experience symptoms from late and long-term effects of treatments such as fatigue, pain, and distress [2]. Concurrently, they must deal with psychosocial stressors such as anxiety, depression, uncertainty about the future, and fear of cancer recurrence as they transition back to their previous roles and responsibilities at home and in the workplace; these factors can impair their quality of life, performance at work, and their ability to contribute to society $[3,4]$. Furthermore, the number of cancer survivors in Canada continues to rise due to rapid advances in early detection and treatments for cancer in an aging population. The most recent data suggest there are over 800,000 Canadians living with a history of cancer diagnosed in the previous 10 years [5]. Moreover, these numbers are expected to increase since survival data indicate $60 \%$ of Canadians with the top 4 most common cancer diagnoses are expected to survive at least 5 years postdiagnosis [6].

Similar trends of rising numbers of cancer survivors have been reported in the United States, and the world over [2]. Thus, cancer survivors globally can benefit from innovative interventions that address their unique psychosocial needs during survivorship. A growing body of evidence supports the efficacy of a range of mind-body therapies in alleviating these and other symptoms in cancer patients and survivors [7]. Among these therapies, mindfulness-based interventions (MBIs) have demonstrated significant efficacy in impacting psychosocial and physical health in the cancer population, such as the Mindfulness-Based Cancer Recovery (MBCR) program, a 9-week group behavioral treatment program that trains participants in mindfulness techniques through meditation and gentle movement practices [8].

The investigators on this research team LC and MS have studied MBCR for the past three decades and have tested its efficacy in a range of studies and groups of people with cancer, with success in impacting a range of biological and psychosocial outcomes including, but not limited to, symptoms of stress, quality of life, and mood disturbance [8-10]. This body of work on MBCR has spanned basic mechanistic research to clinical trials and implementation science. Most research studies of this nature have tested MBIs such as MBCR when delivered face to face in a group-based setting, although a variety of online-based and digitally adapted MBIs are now available to patients through smartphone apps [11]. Mobile app-based MBIs for cancer patients and survivors allow for considerable flexibility and appeal, especially since they eliminate the need for travel time and problems due to scheduling conflicts $[11,12]$.

\section{Digital Health Interventions in Cancer Care}

One of the most significant social and economic changes in the modern world has been the use of computer technology and the internet. Recent data indicate that $76 \%$ of Canadians now own a smartphone device with data connection across all demographic groups, and the numbers are projected to increase consistently $[13,14]$. The popularity of this medium is of great interest for health practitioners, researchers, and policy makers considering the wide-ranging capabilities of smartphone devices. In the field of cancer care, reviews of internet and smartphone app-based interventions for cancer patients and survivors have suggested that cancer patients find these interventions to be highly acceptable and feasible [15,16]. Furthermore, psycho-oncology researchers have advocated for more research with psychosocial interventions that can be delivered using the internet and smartphone apps in the cancer population [15-17].

\section{Benefits of App-Based and Online Mindfulness Interventions}

App-based and online mindfulness-based interventions circumvent problems with traditional face-to-face delivery of MBCR such as work schedules, conflicts with other appointments, lack of childcare, and residing far from treatment centers in remote locations. Another potential benefit of app-based and online mindfulness interventions in cancer care is the considerable cost savings for the health care system without compromising on the quality of care, as online and artificial intelligence technologies can simulate the real-world experiences; in addition, studies have shown these interventions to be highly feasible and acceptable. For example, authors LC and MS conducted a feasibility trial of online MBCR, which found that more than $80 \%$ of participants completed the online MBCR program, and a $10 \%$ response rate to recruitment letters was achieved (the target was 5\%) [18]. Also, recent systematic reviews and meta-analyses of app-based mindfulness interventions in the cancer population report high feasibility and acceptability; albeit engagement is an important variable to consider for intervention success [16,19,20].

However, while there are hundreds of commercially available mindfulness training apps, eg, Headspace, Calm, and 10\% Happier [21,22], only Headspace has been customized for cancer patients and been scientifically evaluated [21]. Data from 
a randomized waitlist-controlled trial and a prospective cohort study of Headspace have demonstrated good overall efficacy for improving outcomes such as quality of life and anxiety in women diagnosed with breast cancer $[21,23]$. However, the overall science of app-based MBIs is still in its early stages and far from achieving consensus about efficacy of intervention platforms, as well as understanding mechanisms of action $[12,17]$. For example, this is demonstrated by a randomized trial of Headspace with college students that reported no effects for the mindfulness component of Headspace's app-based program [24].

Furthermore, Headspace's audio content is exclusively voiced and delivered by the app's founder in a monologue format [25]. This approach may not connect with all cancer patients and survivors, considering the importance of the patient-psychotherapist relationship in determining the success of any psychotherapeutic practice [26,27]. Also, cancer patients and survivors are more inclined to accept and utilize content created and delivered by clinical experts, for reasons such as source credibility and condition-specific content [28-30]. The research team for this study was interested in developing an app-based MBI for cancer survivors that simulated the interactive dialogic approach used in the MBCR program. The research team chose to evaluate the Am Mindfulness app as it could potentially address the gaps in the design and delivery of app-based MBIs for the cancer population.

Am Mindfulness is a readily editable digital platform with audio and visual capabilities and its content can be updated and modified with automatic app updates. Am Mindfulness was developed and is maintained by Mobio Interactive (MI) Inc. [31], a Canadian technology company based in Toronto, Canada, cofounded by MT and BS; user data are maintained on secure servers in Canada and meet security and Health Insurance Portability and Accountability Act privacy standards required for medical data. The name for Am Mindfulness, referred to simply as, $A m$, and pronounced "ahm" was chosen by MI for several reasons. First, "am" is the present tense of the verb "to be," which is a linguistic embodiment of mindfulness given the high emphasis that mindfulness practices place on observing the present moment. Second, because of how "Am" is pronounced, it sounds similar to both the word for "soul" in French ("âme") and the chant "ohm" used in ancient meditative practices from India. Finally, the name "Am" is relatively simple and less likely to bias individuals on the app's purpose or offering in the way that names for other meditation-focused apps such as "Calm" or "Smiling Mind" may.

The design of Am Mindfulness allows for considerable flexibility with delivering content in real time through the "Journeys" app feature. Each app-based "Journey" involves a sequence of custom audio tracks, visuals with text and in-app exercises designed with a particular objective, eg, mindful survivorship. The content of a "Journey" can be seamlessly introduced and refined for a patient population in real time. MI describes their major motivation for creating the "Journeys" feature in Am Mindfulness as their commitment to create and deliver diverse content voiced over by clinical experts, in addition to mindfulness practitioners, to address the needs of specific patient populations [32]. Evidence from a recent randomized trial with a prerelease version of the Am Mindfulness app (called Wildflowers) has demonstrated efficacy evidence for reducing anxiety in college students before a stressful event of an examination [33].

In this project, the Am Mindfulness-Based Cancer Survivorship (MBCS) journey was developed and recorded in the voices of MBCR program facilitators LC and MS. Several audio tracks in the $A m$ MBCS journey have a dialogue and interaction between LC and MS, who are clinical psychologists and leading experts on mindfulness-based cancer recovery, having developed and scientifically evaluated the MBCR program together for over 20 years. Details of the Am MBCS journey are described in the Intervention section and illustrated in Figure 1. In this study, we intend to test the efficacy of the Am MBCS Journey, referred to hereon as $A m \mathrm{MBCS}$, to reduce stress in cancer survivors. As secondary objectives, we will investigate the feasibility of recruitment as well as the usability of the app and in-app data on specific usage patterns. 
Figure 1. The Mindfulness Based Cancer Survivorship (MBCS) Journey Contained within Am. (A) Navigation menu. (B) Core audio content (C) In-app exercises.
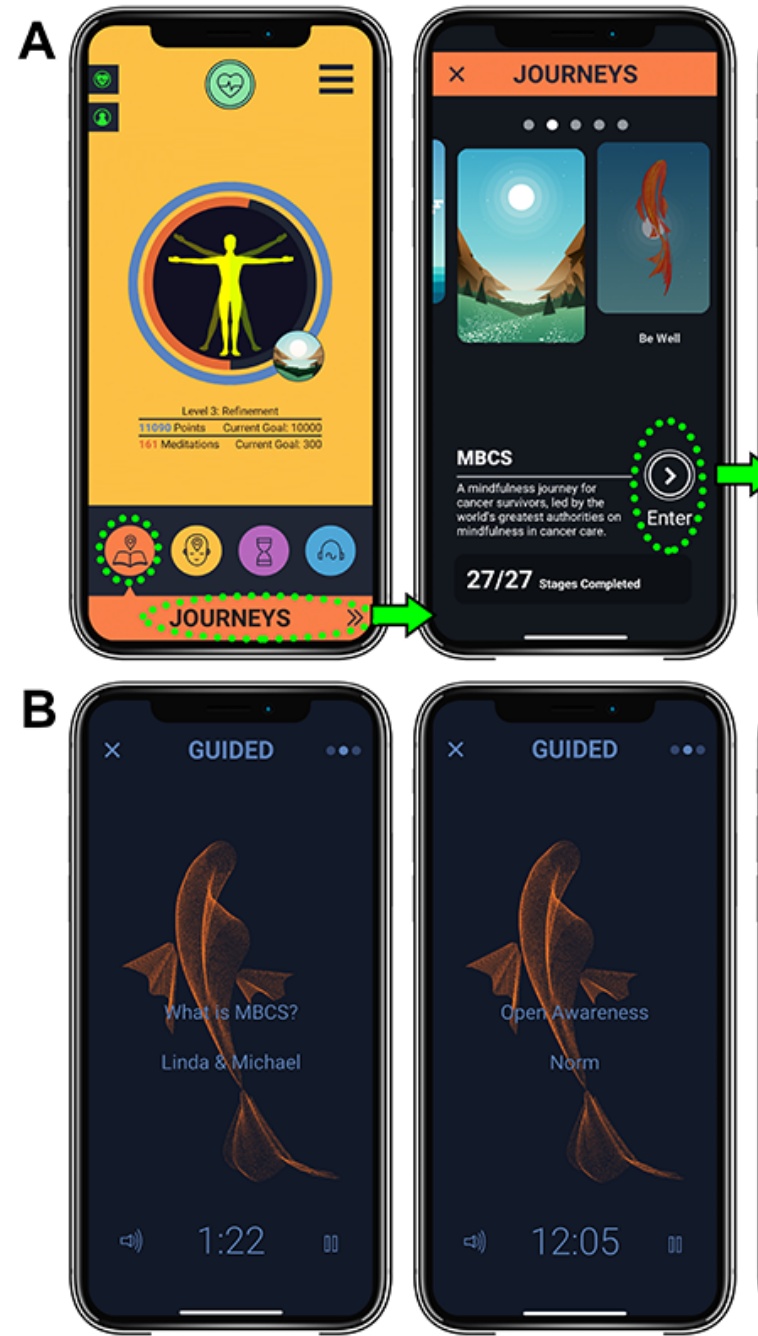

C
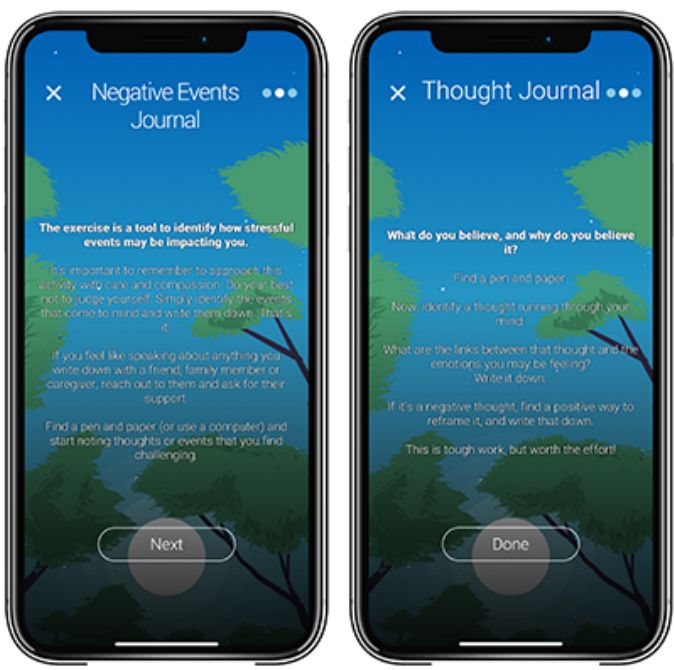

\section{Methods}

\section{Study Design}

The study is a two-armed randomized waitlist-controlled design with 1:1 allocation to treatment (immediate Am MBCS app group) or control (waitlist usual care) arms, with assessments
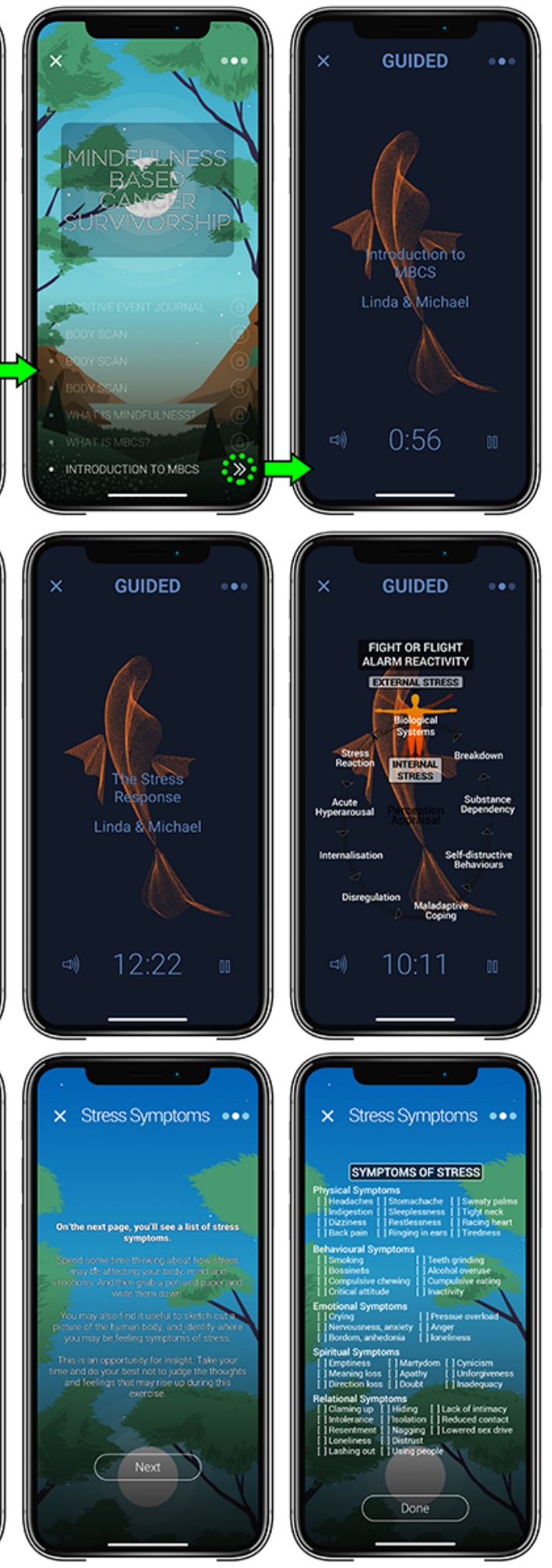

at six time points: (1) baseline, (2) mid-intervention (2 weeks later), (3) immediately postintervention (4 weeks), (4) 3 months postbaseline, (5) 6 months postbaseline, and (6) 12 months postbaseline. A detailed study flowchart is shown in Figure 2. This is an open-label trial, as blinding to interventions is most often not possible in psychosocial intervention research. 
Figure 2. SEAMLESS Study Flow Chart. Includes study design, stage, and all time points of data collection for study assessments.

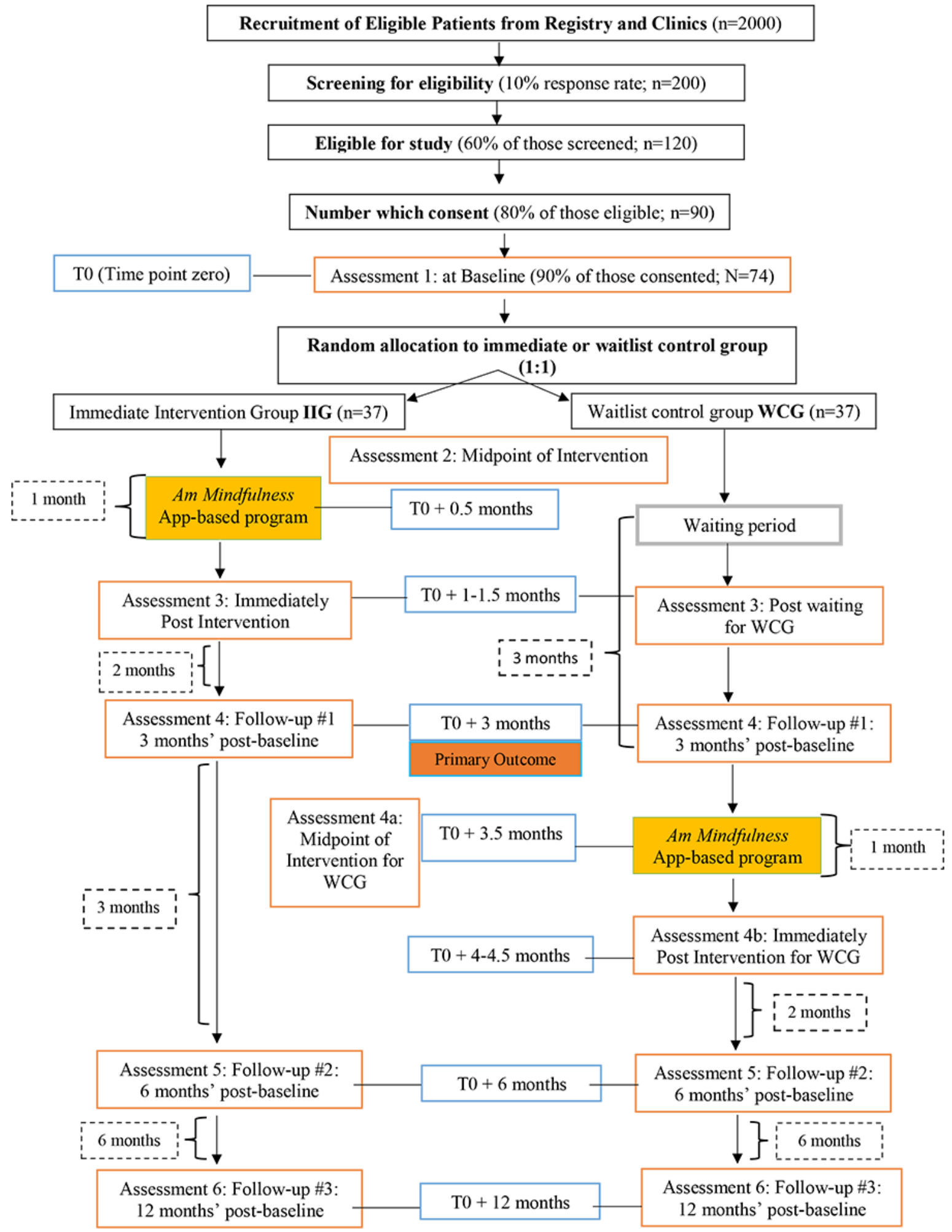

\section{Participants}

Cancer survivors with any type of cancer who have completed their active treatments at least 2 weeks before recruitment in this study will be included. Inclusion criteria are intentionally broad to be pragmatic and improve generalizability to the real world. Refer to Table 1 for detailed rationale for each inclusion and exclusion criterion. 
Table 1. Inclusion and exclusion criteria.

Criteria
Inclusion
(1) Men and women over the age of 18 years
(2) Completed all cancer treatments 2 weeks before
enrollment

(3) Access to a smartphone with data connection

(4) Willing to give time for mindfulness practice

(5) Sufficient ability to speak and read English

(6) Willingness to be randomized into immediate or waitlist groups and complete all assessments

\section{Exclusion}

(1) Suffering from current major depressive disorder, or other psychiatric disorder (self-reported) that would interfere with the ability to participate

(2) Currently engaging in mindfulness meditation one or more times per week

(3) Cognitive impairment that would interfere with completing questionnaires or the intervention; $<6$ on the Brief Screen for Cognitive Impairment (BSCI) [3]
Rationale and notes

Both men and women are included to broaden the generalizability of results and allow sex comparisons. All participants must be adults.

A brief period of time is required for patients to recuperate after their last treatment, before starting a new intervention.

Patients will require access to a smartphone to participate. The study team will communicate primarily by phone, text message, and email. In case some patients' do not have a data plan or an insufficient data plan with their smart phone, we will pay for their data connection (up to $0.5 \mathrm{~GB} /$ month).

Patients need to have the motivation to devote approximately 20 to 30 min daily, which is equal to 5 to 7 sessions a week over the course of 1 month to do the mindfulness meditations and practices.

The audio lectures and meditations and assessments will be conducted in English, so participants must be able to understand the audio and fill out the questionnaires.

People must be comfortable with potentially having to wait to get access to the app-based program for another 3 months, as well as be motivated to give 30 to 40 min of their time to complete the online survey assessments.
Evidence indicates that participants with active psychological disorders should be first treated for these problems individually, before engaging in experimental mental health and meditation programs of this nature, which are not intended to treat these disorders.

To ensure sample homogeneity, the study will include participants who are NOT currently practicing mindfulness, using an app or otherwise. However, this would not exclude everyone who may have casually experimented with the aforementioned interventions in the past.

People require enough cognitive capacity to complete the questionnaires, navigate and listen to the app and complete homework independently. The BSCI only rules out those with significant cognitive impairment and will not exclude those with the milder cognitive impairment associated with cancer-related "brain fog."

\section{Recruitment}

Potential participants will be recruited at a comprehensive cancer center in Western Canada from a Provincial Cancer Registry.

\section{Alberta Cancer Registry}

The Alberta Cancer Registry (ACR) is a population-based registry, established in 1942, that records and maintains data on all new cancer cases and cancer-related deaths occurring in the province of Alberta. The registry records information about the type of cancer and cancer treatments, as well as personal information, such as name, date of birth, sex, provincial health care number, and postal code. The ACR will contact all potential participants on behalf of the research team with a study information letter with the research team's contact information. Potential participants will include patients diagnosed with any type of cancer, who completed their treatments at least 2 weeks prior and who reside outside the Calgary metropolitan region. The geographical criteria were chosen to access cancer survivors in urban and rural areas of Alberta that are distant from the University Health Center, as most mindfulness-based programs and studies have been mainly accessible for residents of the Calgary metropolitan region due to its proximity to the University Health Center. The ACR's method of contact ensures patient privacy and provides patients the choice to participate in the study. Interested participants will then contact the research team, and then are further screened for inclusion criteria.

\section{Sample Size}

In previous studies with face-to-face group MBCR, we have observed medium effects for symptoms of stress (measured by the Calgary Symptoms of Stress Inventory [C-SOSI]) as the primary outcome and expect similar effects in this study. Also, recent meta-analyses of MBIs and psychosocial stress outcomes have demonstrated a similar medium standardized effect size of Cohen $d=0.5$ for stress [34]; also, most MBI studies collect their primary outcome at 3 months postbaseline as most mindfulness interventions span 6 to 10 weeks [7,34]. For this study, we therefore used a medium effect size $(f=0.25)$ to conduct an a priori sample size calculation using the software $\mathrm{G}^{*}$ Power 3.1.9.3 developed and maintained by researchers at Heinrich Heine University Düsseldorf [35]. The other parameters for estimating the sample size included, a standard type- 1 error rate, $\alpha=.05$, and a low risk of type- 2 error, power $(1-\beta)=.95$, to detect interaction effects between time points (baseline and 3 months postbaseline) and group (intervention and control) using a repeated measures analysis of variance with correlation among repeated measures assumed to be 0.5 and nonsphericity correction $\varepsilon=1$. The total required sample size reported by $\mathrm{G}^{*}$ Power was 54 participants to detect such an interaction effect.

In addition, based on our previous experience with online and in-person MBI trials and previous app-based studies $[10,18,20,34,36]$, we assumed approximately $20 \%$ attrition and 
$10 \%$ to $15 \%$ probability of missing data. Therefore, we oversampled for this study accordingly. Hence, the final total sample size we aim to recruit in this study is $\mathrm{N}=74$, with $\mathrm{n}=37$ in the immediate intervention arm and $n=37$ in the waitlist control arm. Please refer to Figure 2 for the study flowchart that describes estimated sample size calculations at each assessment.

\section{Randomization}

Participants will be randomized by the study statistician, by generating participant ID numbers and group allocations for the entire study in advance using a random number generator program in SPSS. Block lists of randomized participant IDs will then be uploaded to the Research Electronic Data Capture (REDCap) randomization module, which will allow the study staff to provide immediate group allocation to participants after completion of consent procedures. Only the study statistician will develop the group assignments, which are locked by REDCap after upload, to prevent selection bias. Randomization will occur after the baseline assessment, and those in the immediate Am MBCS intervention group will get a text message and email containing the link to download the Am Mindfulness app, from the Android or Apple app store. The waitlist control group will be informed that they will need to wait for the intervention and will be contacted when they can download the AmMindfulness app and start the intervention.

\section{Informed Consent Procedures}

Informed consent will be obtained electronically through the secure, web-based app designed to support data capture for research studies, REDCap, which is supported by the technology team at the University of Calgary, Canada, where this research is being conducted. REDCap's web-based app uses secure two-factor web authentication, data logging, and secure sockets layer encryption that ensures the security and confidentiality of private information for obtaining informed consent [37]. An email with a survey link will be sent to the participant. After the participant clicks on the link for the study from their email client, the first page of the REDCap survey for this study will open in a new window or tab and will contain the details of the informed consent form. Participants will check the box "Yes" that asks them whether they completely understood the terms of their voluntary participation in the study.

Participants will then actively provide electronic consent to the study by clicking on the "Agree" button, which will be preceded by stating that, "Clicking on the 'Agree' button below indicates that (1) you have read the informed consent information, (2) you voluntarily agree to participate, and (3) you are at least 18 years of age." Participants will also have the option to opt out of the study by clicking the "Disagree" button, which will be followed by the statement, "If you do not wish to participate in the research study, please decline participation by clicking on the 'Disagree' button."

Only those participants that click on "Agree" will be able to proceed with completing the rest of the questionnaires. Participants will be able to download and save a PDF version of the consent form for their records. Participants will enter their name, email, and cellphone number after completing the form. After participants provide their online consent, they will be asked to complete the baseline measures online on REDCap. Subsequently, participants will be sent a study welcome email, which will contain orientation material and instructional pictures and videos about how to use the Am app, and instructions for the 4-week intervention. In addition, the study staff will also conduct a study orientation phone call to guide participants to using the app with ease.

\section{Intervention}

\section{The Am Mindfulness-Based Cancer Survivorship Journey}

Am Mindfulness ("Am"; second generation of the app, Wildflowers Mindfulness) supports a personalized mindfulness practice through guided meditations, audio lectures and discourses, reminders, a timer to facilitate self-guided meditation, journaling features, and psychobiometric recordings and feedback. The Am app is currently available in four languages (English, Mandarin, Dutch, and German) and can be viewed and downloaded from the Android and Apple app stores with the platform-agnostic link [38]. Within Am, study participants will be instructed to access the Am MBCS journey. $A m$ MBCS has a total of 27 steps that include, audio-recorded lectures, guided meditations such as body scans, and writing an events journal; see Figure 1 to screenshots of the Am MBCS journey contents. The curriculum is based on our previous experience with the evidence-based MBCR program and related meditations, which is described under 5 discrete units of learning in Table 2. The content of the audio instruction by LC and MS is similar to that delivered in the in-person classes on these units, with examples specific to situations and symptoms common for people living with cancer. Common topics such as coping with pain, insomnia and fear of cancer recurrence are included.

Participants will be encouraged to participate in the app-based activities for 20 to 30 min every day, with a minimum of 4 days in a week, over a period of 4 weeks. To promote engagement with the app-based program, user data will be tracked confidentially (see the section on Feasibility, Acceptability, Adherence, and Contamination) and users with low engagement will be sent text messages to promote app usage, eg, "Please remember to listen to your Am MBCS mindfulness recordings for today." Text messages will be delivered using the secure communications platform, Twilio [39]. Also, the research team will monitor participant engagement metrics on a daily basis, and low or nonengaged users will receive a phone call with support for problem solving. In addition, Am Mindfulness also sends users regular push notifications, which appear as motivational messages on the user's mobile screen that may also promote engagement.

Patients using the app can also access the meditations on Am through the app's "Library" feature, which contains all the meditations on the app indexed by author and name. Although some meditations are perpetually free, users need to pay for the full selection of guided sessions and biofeedback technology; therefore, participants in this study will be provided with a 12-month paid subscription to the Am app. 
Table 2. App-based mindfulness-based cancer survivorship curriculum.

\begin{tabular}{ll}
\hline Unit \# & Topics or focus of module \\
\hline 1 & $\begin{array}{l}\text { What is mindfulness; why mindfulness for cancer?; belly breathing } \\
\text { exercise; introduction to Body Scan with focus on cancer-related } \\
\text { changes in the body. }\end{array}$ \\
2 & $\begin{array}{l}\text { Mindful attitudes (nonjudgment, acceptance, nonattachment) in } \\
\text { the context of cancer. }\end{array}$ \\
3 & $\begin{array}{l}\text { Stress response; biology of stress, stress and cancer; link between } \\
\text { inner narrative and chronic stress; sleeping well exercise. }\end{array}$ \\
4 & $\begin{array}{l}\text { Stinkin' Thinkin'; maladaptive stories we tell ourselves; common } \\
\text { cognitive distortions with cancer-related examples; coping with } \\
\text { thoughts and fears of cancer recurrence. }\end{array}$ \\
& $\begin{array}{l}\text { Introduction to guided imagery; using imagery to cultivate loving } \\
\text { kindness toward the suffering of self and others. }\end{array}$ \\
\hline
\end{tabular}

\section{Am's App-Based Stress Measurement}

The Am app also has four innovative stress measurement features that serve as exploratory outcomes for this trial. First, cognitive stress is objectively quantified via a 30 -second "selfie" video that uses an algorithm to extract heart rate and heart rate variability from the biosignals inherent to the human face using photoplethysmographic imaging principles [40]. The amount of cognitive stress is determined via deep neural networks trained on tens of thousands of video and stress pairings and has reported $86 \%$ accuracy for determining an individual's stress as "very low," "medium low," "medium high," and "very high" [41]. Second, emotional stress levels are obtained via a digital 4-quadrant emotion mapping board (mood board) that lists emotions such as "happy," "sad," and "tense" ranging on one axis from unpleasant to pleasant, and along another axis from mild to intense; see Figure 3 for screenshots of the in-app stress assessments. Each emotion listed within the mood board is associated with a score that is not disclosed to the user and used to calculate mood. Third, subjective stress is obtained via a slider ranging from "none" to "extreme." Fourth, personal notes are input using an open field text box. The output of the mood board and stress slider provides data that have been benchmarked to standard psychological surveys [33].

The Am app uses secure web authentication, data logging, and encryption that ensures security and confidentiality of any personal identifiable information and in-app data. Am and its previous version Wildflowers have together received approximately 100,000 downloads and $\mathrm{Am}$ is rated 4.8 stars from 23 reviews in the Canadian Apple app store as of July 30, 2019.

Figure 3. In-App Psychobiometric Assessments within Am. (A) "Selfie" video using photoplethysmography technology to quantify stress; (B) "Mood board" containing 32 emotion words and "stress slider" for stress self-assessment; and (C) "Journaling" feature to input experiences.

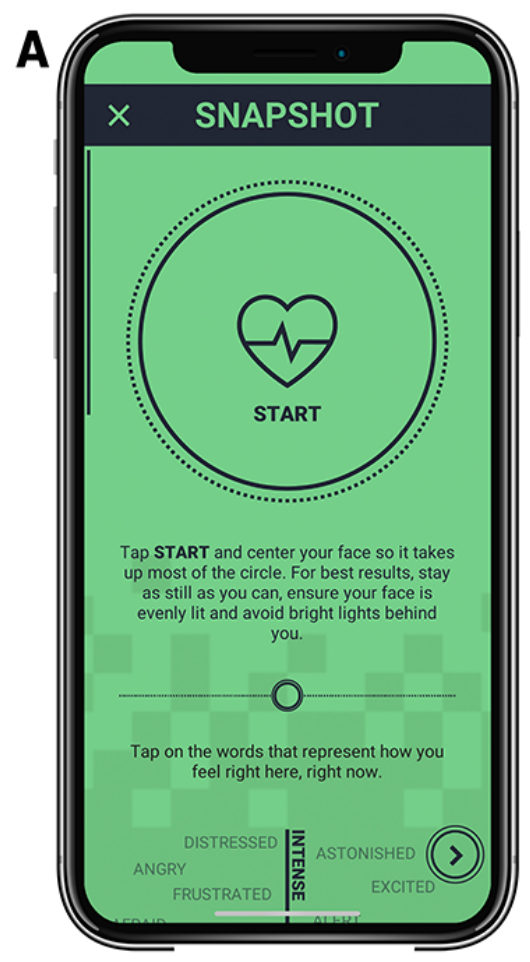

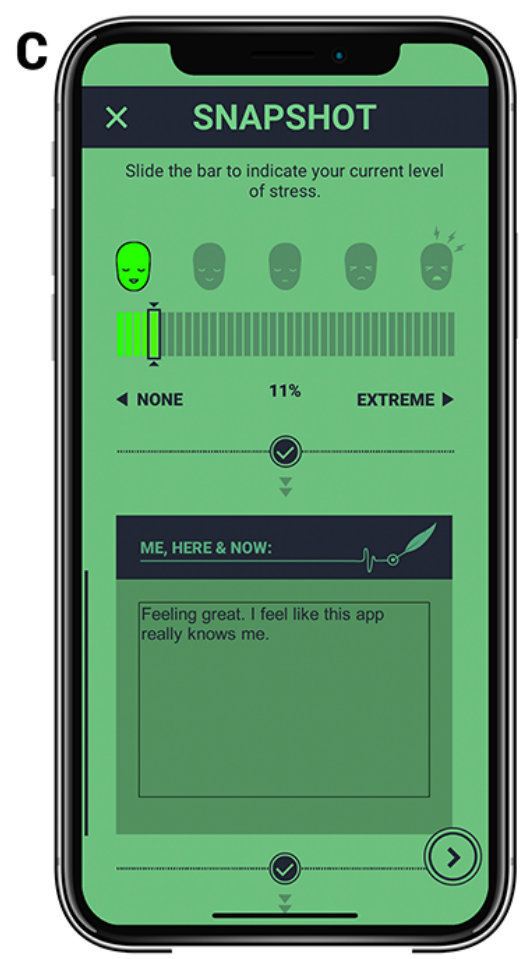




\section{Procedures}

\section{Experimental Group}

Participants will be provided a 12-month paid subscription to $A m$. The first month of this Am subscription is dedicated to the study intervention. Users will be encouraged to participate in the app-based activities for 20 to 30 min every day, with a minimum of 4 days in a week, after which they will get reminder notifications.

\section{Waitlist Control Group}

Participants will receive treatment as usual, followed by a delayed (waitlist) intervention of the same Am MBCS app-based intervention after the 3-month postbaseline assessment of the $A m$ app intervention group (see Figure 2). Control group participants will get access to the Am app only after completing their 3-month waiting period.

\section{Trial Registration and Reporting}

This trial has been registered at the ClinicalTrials.gov database of privately and publicly funded clinical studies [42]. The results will be reported as per the updated Consolidated Standards of Reporting Trials (CONSORT) eHealth checklist [43] version 1.6.1. and will follow guidelines and the flow diagram for reporting nonpharmacological treatments [44].

\section{Outcome Measures}

The outcome measures employed in this study include a series of well-validated psychometric instruments for assessing a variety of psychosocial constructs. See Table 3 for a detailed description of outcomes. We will also use the standardized outcome measures available from the Patient-Reported Outcomes Measurement Information System (PROMIS), which is a set of person-centered measures that evaluate physical and psychosocial health in adults and children [45]. The advantage of using PROMIS measures is that they are psychometrically sound and have been created to be relevant across all conditions for the assessment of symptoms and functions [45]. Finally, we are also going to obtain and analyze the data from the Am app with regard to the user's self-report, biometrics, and engagement. The entire battery of questionnaires will be completed securely online and requires approximately 30 to $45 \mathrm{~min}$. 
Table 3. Outcome measures.

\begin{tabular}{lll}
\hline Construct & Measure (abbreviation) & Description \\
\hline Screening measures & $\begin{array}{l}\text { Brief Screen for Cognitive Im- } \\
\text { pairment (BSCI) [46] }\end{array}$ & $\begin{array}{l}\text { The BSCI consists of } 3 \text { items which are asked to the patient over the phone. The first } \\
\text { item on the BSCI consists of a memory recall question, and the other } 2 \text { items ask } \\
\text { about ability to carry out daily tasks without help. The scores obtained from the } 3 \\
\text { items are then weighted and summed to arrive at the final BSCI score wherein }>6 \text { is } \\
\text { significant impairment. }\end{array}$
\end{tabular}

\section{Background measures}

Demographics and medical history

\section{Primary outcome}

Symptoms of stress

Age, sex, marital status, education, other medical conditions, and medications

Calgary Symptoms of Stress Inventory (C-SOSI) [47]

\section{Secondary outcomes}

Fear of cancer recurrence

Fear of Cancer Recurrence Inventory (FCRI) [48]

Mindfulness

Rumination

Rumination-Reflection Questionnaire (RRQ) [50]

Experiential avoidance

Acceptance and Action Questionnaire (AAQ) [51]

Anxiety

Patient-Reported Outcomes Measurement Information System (PROMIS)-Cancer Bank v 1.0-Anxiety [52]
Mindfulness Attention Awareness Scale (MAAS) [49]
Age, sex, marital status, education, other medical conditions, and medications. All these constructs will be assessed using standardized self-report items.
The C-SOSI is a 56-item scale, derived from exploratory factor analysis on the 95item Symptom of Stress Inventory (SOSI) collected from cancer patients who attended our MBCS program. A 5-point scale ("never" to "very frequently") is used to rate the frequency of stress-related symptoms in the past week. There is a total score and 8 subscales (depression, anger, muscle tension, cardiopulmonary arousal, sympathetic arousal, neurological or GI, cognitive disorganization, and upper respiratory symptoms), all of which have high internal consistency ( 0.80 to 0.95 ), and the total score has good convergent and divergent validity with other well-validated measures.

FCRI contains 42 items, evaluating 7 components associated with the fear of cancer recurrence: triggers, severity, psychological distress and functioning impairments, insight scale, reassurance, and coping strategies. Each item is measure one a Likert scale ranging from 0 (not at all or never) to 4 (a great deal or all the time). Total score can be obtained from each subscale and a total FCRI score can be obtained by adding the total scores of all subscales, higher scores indicate higher levels of fear of cancer recurrence.

MAAS is a 15 -item scale, designed to assess characteristics associated with mindfulness, such as open or receptive awareness of and attention to what is taking place in the present. Participants use a scale from 1 to 6 (almost always to almost never), to indicate how frequently or infrequently they have each experience. Higher scores reflect higher levels of dispositional mindfulness. A thorough validation process has demonstrated the reliability and validity of the MAAS with high internal consistency, $\alpha=.86$.

The RRQ is a 24-item, 5-point Likert Scale. The rumination subscale of the RRQ assesses recurrent, primarily past-oriented thinking about the self, which is prompted by threats, losses, or injustices to the self. The scale correlates with mindfulness in expected directions and has demonstrated high internal consistency of $\alpha=.92$.

The AAQ was developed to measure experiential avoidance, the tendency to negatively evaluate internal experiences. (eg, emotions and body sensations), unwillingness to be in contact with such experiences, and the need to control or alter them or the contexts that engender them [51]. The psychometric properties of versions of the AAQ have been well established in clinical (eg, anxiety disorder) and nonclinical samples. The 16-item AAQ that will be used in this study produces a single factor, with acceptable internal consistency, $\alpha=.77$.

PROMIS-Anxiety questionnaire assesses the anxiety domains of self-reported fear (fearfulness, panic), anxious misery (worry, dread), hyperarousal (tension, nervousness, restlessness), and somatic symptoms related to arousal (racing heart, dizziness). All PROMIS-Cancer instruments were developed for use with any cancer patient. The PROMIS-Cancer Anxiety item bank contains a total of 22 items, 20 of which are also in the PROMIS-Anxiety item bank, so it can be correlated with studies of other clinical populations. The PROMIS-Cancer Anxiety item bank will be delivered to patients in this study. The PROMIS-Cancer Anxiety has demonstrated high internal consistency (Cronbach $\alpha>$.9). 


\begin{tabular}{|c|c|c|}
\hline Construct & Measure (abbreviation) & Description \\
\hline Depression & $\begin{array}{l}\text { PROMIS-Cancer Bank } \\
\text { v1.0-Depression [53] }\end{array}$ & $\begin{array}{l}\text { PROMIS-Depression questionnaire for cancer patients assesses the domains of de- } \\
\text { pression, which include self-reported negative mood (sadness, guilt), views of self } \\
\text { (self-criticism, worthlessness), and social cognition (loneliness, interpersonal alien- } \\
\text { ation), as well as decreased positive affect and engagement (loss of interest, meaning, } \\
\text { and purpose). Somatic symptoms (changes in appetite, sleeping patterns) are not in- } \\
\text { cluded. The PROMIS-Cancer Depression item bank contains a total of } 30 \text { items, } 23 \\
\text { of which are also in the PROMIS-Depression item bank, so it can be correlated with } \\
\text { studies of other clinical populations. The PROMIS-Cancer Depression item bank } \\
\text { will be delivered to patients in this study. The PROMIS-Cancer Depression has } \\
\text { demonstrated high internal consistency (Cronbach } \alpha>9 \text { ). }\end{array}$ \\
\hline
\end{tabular}

Fatigue

PROMIS-Cancer Bank v1.0-Fatigue [54]

Physical Function

PROMIS-Cancer Bank v1-Physical Function [55]

Return to work

Exploratory measures

User self-report

Mood, stress, and intent for mindfulness

Employment, hours of paid work, ability to work, and rate of return-to-work at 12 -months

(est

PROMIS-Cancer Fatigue measure assesses a range of self-reported symptoms from mild subjective feelings of tiredness to an overwhelming, debilitating, and sustained sense of exhaustion that likely decreases one's ability to execute daily activities and function normally in family or social roles. Fatigue is divided into the experience of fatigue (frequency, duration, and intensity) and the impact of fatigue on physical, mental, and social activities. The PROMIS-Cancer Fatigue item bank contains a total of 54 items, all of which are also in the PROMIS-Fatigue item bank and will be delivered to patients in this study. The PROMIS-Ca Fatigue has demonstrated high internal consistency (Cronbach $\alpha>9$ ) in numerous studies within cancer and other clinical populations [54].

PROMIS-Physical Function instruments measure self-reported capability rather than actual performance of physical activities. This includes the physical functioning, mobility as well as instrumental activities of daily living, such as running errands. The PROMIS-Cancer Physical Function has items specific to cancer patients and survivors. The PROMIS-Cancer Physical Function item bank contains a total of 45 items, 33 of which are also in the PROMIS-Physical Function item bank [55], and will be delivered to patients in this study. The PROMIS-Cancer Physical Function has demonstrated high internal consistency (Cronbach $\alpha>$.9).

Self-reported work status will be assessed at each time point including (1) current working status (working full-time; part-time; retired; short- and long-term disability; unpaid homemaker); (2) weekly hours of paid work; and (3) job type using a wellestablished job classification system. If applicable, participants will be asked at followup on what date they returned to paid work.

Stress: Adjusting a dynamic slider between the minimum score "no stress" and the maximum score "max stress."

Mood Board: Participant can select between 1 and 24 "mood words" that indicate how they are feeling, eg, angry, happy, elated, and sad.

User biometrics
Photoplethysmographic imaging, which is the measurement of volumetric change observed via the selfie camera of the smartphone, provides data that can be used to infer user biometrics, such as heart rate, respiratory rate, and relative blood oxygen saturation.

\section{Feasibility, Acceptability, Adherence, and Contamination}

\section{Feasibility of Intervention}

The ACR provides the specific number of potential participants contacted for the study across the province. The number of participants who were invited through the registry, those who contact the team showing interest, as well those screened for eligibility, completion of intervention, and each assessment point will be tracked (see Figure 2). Intervention response rate and overall completion rate will be calculated from the aforementioned data points.

\section{Acceptability, Adherence, and Engagement}

The app usage of patients will be tracked through the engagement data from the app, which include session length, identity, type and frequency, points and badges earned, number of page and screen views, mindful activities in the app, total time spent on the mindfulness audio tracks, and number of daily visits to the app. Am also records which lessons and meditations participants accessed frequently. Participants with low engagement during the $A m$ MBCS intervention period, defined as less than 4 times a week, will get reminder notifications through text message and phone calls with resources for problem solving. Acceptability and adherence to the intervention will be estimated from the engagement obtained from the app and completion rate of outcome assessments

\section{Contamination}

A standardized form assessing the use of a range of complementary therapies will be administered at each time point. We will also ask the waitlist control group if they used any other mindfulness or meditation apps during their 3-month waiting period. 


\section{Objectives and Hypotheses}

\section{Objective 1: Primary Outcome}

The first objective is to evaluate the efficacy of the $A m$ app-based MBCS program to relieve symptoms of stress (primary outcome).

Hypothesis 1: Compared with controls, the Am app-based MBCS program participants will report significantly less symptoms of stress at 3 months postbaseline (primary outcome) assessment.

\section{Objective 2: Secondary Outcomes}

The second objective is to evaluate the efficacy of the Am app-based MBCS program to decrease the fear of cancer recurrence, anxiety, depression, and fatigue, and to improve overall physical functioning (secondary outcomes) at 3 months postbaseline assessment.

Hypothesis 2: Compared with controls, the Am app-based MBCS program participants will report significantly less fear of cancer recurrence, rumination, experiential avoidance, anxiety, depression, and fatigue, and increased mindfulness and overall physical functioning at 3 months postbaseline (secondary outcomes).

\section{Objective 3: Exploratory Outcomes}

This includes the exploratory aims as follows: (1) to explore correlations between the self-reported outcome data and the psychobiometric data collected by the Am app, (2) to determine changes over time between the Am app-based MBCS program and the waitlist control group.

Hypothesis 3: Self-reported data obtained from all participants will significantly correlate with the app-based self-reported stress data and biometric stress data.

Hypothesis 4: To determine the short-term, medium-term, and long-term effects of the Am app-based MBCS program with regard to the primary and secondary outcomes collected at all time points.

\section{Data Analysis}

Participants will enter data from their home computers or smartphones using the secure REDCap data collection and management system (approved by the University of Calgary and Alberta Health Services). Data will then be transferred into SPSS and or SAS for analysis. Data analyses will utilize linear mixed models (LMM) and intent-to-treat (ITT) principles to assess several planned comparisons across the groups based on identified aims and hypotheses.

\section{Intent-to-Treat Analysis}

Data related to recruitment, participation, and dropout rates will be reported according to the guidelines given by the CONSORT-eHealth statement [43]. All participants that entered our study will be included in our analyses and will be retained in the arm (treatment or control) to which they were originally randomly allocated. This study will employ an ITT analysis design, wherein participants who were nonadherent to the protocol will be included in statistical analyses, regardless of their alignment with the inclusion criteria, the treatment they received, and if they withdrew from the intervention protocol (attrition) completely or deviated from the protocol (nonadherence).

\section{Descriptive Statistics}

Data will be cleaned, the descriptive statistics of the sample will be assessed, and all variables will be checked for normality of distribution. Descriptive statistics of our sample will be calculated to summarize demographic and disease-related characteristics and check for group differences between groups using Chi-squared and $t$ tests. In case data are nonnormal, the Kenward-Roger correction for degrees of freedom will be applied to the LMM. Potential treatment moderators of age, sex, cancer type, cancer stage, and chemotherapy regimen will also be included as possible covariates. We will also conduct correlation and regression analyses to determine correlations between scores of the primary and secondary outcome measures, eg, correlation between C-SOSI and PROMIS-Cancer Anxiety scores, and between secondary outcome measures, eg, correlation between PROMIS-Cancer Fatigue scores and mood words selected by the participant in the Am app.

\section{Hypothesis Testing}

\section{Hypothesis 1}

LMM is a suitable statistical method for this study because of the ability to perform sophisticated statistical imputation of data missing at random in a longitudinal study design. In addition, the LMM also includes mixed effect methods with a random intercept model, which can account for the variances between participants and within participants. Therefore, we plan to use the LMM analyses for testing hypotheses 1 and 2, wherein the LMM will estimate differences between the immediate group and waitlist control group by conducting a group $\times$ time interaction analysis with a significance level of $\alpha<.05$. Each of the LMMs will include fixed effects for time (within-subjects factor) and group (between-subjects factor) and a random effect for the participant. Also, the restricted maximum likelihood estimate method in the LMM will be used to estimate the model parameters and standard errors with a compound symmetry covariance structure to account for the correlation between measurements. Data for testing hypothesis 1 will be C-SOSI total scores and subscale scores for 3 months postbaseline. Within- and between-group differences for the immediate and waitlist groups revealed by the LMMs will be reported with respective $P$ values and the specific model effects, $F(d f)$.

\section{Hypothesis 2}

Similar to hypothesis 1 , for hypothesis 2 , we will use the same LMMs to test for within- and between-group differences for the secondary outcomes.

\section{Hypothesis 3}

For hypothesis 3, linear and curvilinear multiple regression models will be used, along with simple Pearson correlations to detect associations between the primary and secondary self-reported outcomes and the exploratory outcomes obtained from the app data. 


\section{Hypothesis 4}

The short-term (2 weeks and 1 month postbaseline), medium-term (3 and 6 month postbaseline), and long-term (6 and 12 month postbaseline) longitudinal changes in the primary and secondary outcomes will be determined using the LMM quadratic model regressions. To account for the correlation between measurements, the restricted maximum likelihood estimate method in LMM will be used to estimate the model parameters and standard errors with a compound symmetry covariance structure. In addition, analyses with data nesting within participants will also be conducted that will control for the invariant part of each participant's scores. The LMM regression weights $(\beta)$ as well LMM regression coefficients will be reported along with a quadratic regression graph including all time points of data collection.

\section{Results}

Recruitment commenced in January of 2019 and the target sample for enrollment was reached on May 2, 2019. Currently 83 patients have consented and enrolled in the study and are in various stages of their assessments and programs. Anticipated date for the completion of primary outcome data collection is August 1, 2019. Also, data collection for the entire trial is expected to be completed by May 2020.

\section{Discussion}

\section{Limitations}

Considering app-based mindfulness interventions in cancer care are still in the early stages of design and testing, this study has certain design- and intervention-related limitations. First, regarding study design-related limitations, this trial included survivors of all cancer types and stages, which results in high levels of variability of symptoms and cancer-related side effects, which may impact the internal validity of the trial and mask treatment-related effects because of the intervention. However, as the ultimate aim of this research is to reach all cancer survivors regardless of geography, the inclusion criteria were intentionally kept broad to mirror the real-world usage. Second, in terms of the intervention, we selected a 4-week duration for the app-based mindfulness program based on a similar app-based study of a previous version of Am Mindfulness, called Wildflowers, with anxious college students [33]. This 4-week length of the app-based program may not be long enough for cancer survivors, especially those who are completely new to mindfulness meditation and related stress management techniques. Indeed, in-person mindfulness programs for cancer survivors have been between 6 and 9 weeks $[8,11,56]$. Future research into dose-response efficacy of app-based mindfulness interventions in the cancer population is needed to provide an evidence-based duration for app-based mindfulness programs in cancer care.

\section{Conclusions}

This study has the potential to provide a large-scale delivery tool for mind-body therapies to effectively reach cancer patients and survivors the world over. Cancer patients are often unable to successfully participate in face-to-face group programs for a variety of reasons. A smartphone app-based mindfulness program can overcome several difficulties faced by cancer survivors with participating in mindfulness interventions. Patients can participate from home in real time without the added burden of travel, parking, and walking to classes. If effective, this type of low-cost, mobile app-based intervention would be readily welcomed by patients and could easily be translated into clinical practice to reach a large number of patients and survivors, no matter where they reside, including those in remote locations.

\section{Acknowledgments}

This study is funded by the Enbridge Research Chair in Psychosocial Oncology and a Canadian Institutes of Health Research, Strategy for Patient Orientated Research Mentorship Chair in Innovative Clinical Trials, awarded to LEC.

\section{Conflicts of Interest}

Two coinvestigators on this project, MT and BJS, are the cofounders of MI and are majority shareholders of the company at approximately $40 \%$ each. One coinvestigator, NASF, is an advisor for MI and holds <1\% of the company's stock. The remaining authors declare no conflicts of interest.

\section{References}

1. Easley J, Miedema B, Carroll JC, O'Brien MA, Manca DP, Grunfeld E. Patients' experiences with continuity of cancer care in Canada: Results from the CanIMPACT study. Can Fam Physician 2016 Oct;62(10):821-827 [FREE Full text] [Medline: 27737982]

2. National Research Council, Institute of Medicine, Ganz PA, Hewitt M. From Cancer Patient to Cancer Survivor: Lost in Transition. Washington, DC: National Academies Press; 2006.

3. Leach CR, Weaver KE, Aziz NM, Alfano CM, Bellizzi KM, Kent EE, et al. The complex health profile of long-term cancer survivors: prevalence and predictors of comorbid conditions. J Cancer Surviv 2015 Jun;9(2):239-251. [doi: 10.1007/s11764-014-0403-1] [Medline: 25319681]

4. Ward E, Sherman R, Henley S, Jemal A, Siegel D, Feuer E, et al. Annual Report to the Nation on the Status of Cancer, featuring cancer in men and women age 20-49 years. J Natl Cancer Inst 2019 Dec 1;111(12):1279-1297 [FREE Full text] [doi: 10.1093/jnci/djz106] [Medline: $\underline{31145458]}$ 
5. Cuthbert CA, Samawi HH, Hemmelgarn BR, Cheung WY. Effectiveness and components of self-management interventions in adult cancer survivors: a protocol for a systematic review and planned meta-analysis. Syst Rev 2018 Dec 20;7(1):238 [FREE Full text] [doi: 10.1186/s13643-018-0902-7] [Medline: 30572935]

6. Ellison LF. Progress in net cancer survival in Canada over 20 years. Health Rep 2018 Sep 19;29(9):10-18 [FREE Full text] [Medline: 30230521$]$

7. Hall DL, Luberto CM, Philpotts LL, Song R, Park ER, Yeh GY. Mind-body interventions for fear of cancer recurrence: A systematic review and meta-analysis. Psychooncology 2018 Nov;27(11):2546-2558 [FREE Full text] [doi: 10.1002/pon.4757] [Medline: 29744965]

8. Carlson LE. Mindfulness-based interventions for coping with cancer. Ann N Y Acad Sci 2016 Jun;1373(1):5-12. [doi: 10.1111/nyas.13029] [Medline: 26963792]

9. Carlson L, Tamagawa R, Stephen J, Doll R, Faris P, Dirkse D, et al. Tailoring mind-body therapies to individual needs: patients' program preference and psychological traits as moderators of the effects of mindfulness-based cancer recovery and supportive-expressive therapy in distressed breast cancer survivors. J Natl Cancer Inst Monogr 2014 Nov;2014(50):308-314. [doi: 10.1093/jncimonographs/lgu034] [Medline: 25749597]

10. Carlson LE, Doll R, Stephen J, Faris P, Tamagawa R, Drysdale E, et al. Randomized controlled trial of Mindfulness-based cancer recovery versus supportive expressive group therapy for distressed survivors of breast cancer. J Clin Oncol 2013 Sep 1;31(25):3119-3126. [doi: 10.1200/JCO.2012.47.5210] [Medline: 23918953]

11. Lengacher CA, Reich RR, Ramesar S, Alinat CB, Moscoso M, Cousin L, et al. Feasibility of the mobile mindfulness-based stress reduction for breast cancer (mMBSR(BC)) program for symptom improvement among breast cancer survivors. Psychooncology 2018 Feb;27(2):524-531. [doi: 10.1002/pon.4491] [Medline: 28665541]

12. Crandall A, Cheung A, Young A, Hooper AP. Theory-based predictors of mindfulness meditation mobile app usage: a survey and cohort study. JMIR Mhealth Uhealth 2019 Mar 22;7(3):e10794 [FREE Full text] [doi: 10.2196/10794] [Medline: $\underline{30900992]}$

13. Matthew-Maich N, Harris L, Ploeg J, Markle-Reid M, Valaitis R, Ibrahim S, et al. Designing, implementing, and evaluating mobile health technologies for managing chronic conditions in older adults: a scoping review. JMIR Mhealth Uhealth 2016 Jun 9;4(2):e29 [FREE Full text] [doi: 10.2196/mhealth.5127] [Medline: 27282195]

14. Statistics Canada. The Internet and Digital Technology URL: https://www150.statcan.gc.ca/n1/pub/11-627-m/ 11-627-m2017032-eng.htm [accessed 2019-07-01]

15. Leykin Y, Thekdi SM, Shumay DM, Muñoz RF, Riba M, Dunn LB. Internet interventions for improving psychological well-being in psycho-oncology: review and recommendations. Psychooncology 2012 Sep;21(9):1016-1025 [FREE Full text] [doi: 10.1002/pon.1993] [Medline: 21608075]

16. Jongerius C, Russo S, Mazzocco K, Pravettoni G. Research-tested mobile apps for breast cancer care: systematic review. JMIR Mhealth Uhealth 2019 Feb 11;7(2):e10930 [FREE Full text] [doi: 10.2196/10930] [Medline: $\underline{30741644]}$

17. Osborn J, Ajakaiye A, Cooksley T, Subbe CP. Do mHealth applications improve clinical outcomes of patients with cancer? A critical appraisal of the peer-reviewed literature. Support Care Cancer 2020 Mar;28(3):1469-1479 [FREE Full text] [doi: 10.1007/s00520-019-04945-4] [Medline: 31273501]

18. Zernicke KA, Campbell TS, Speca M, McCabe-Ruff K, Flowers S, Carlson LE. A randomized wait-list controlled trial of feasibility and efficacy of an online mindfulness-based cancer recovery program: the eTherapy for cancer applying mindfulness trial. Psychosom Med 2014 May;76(4):257-267. [doi: 10.1097/PSY.0000000000000053] [Medline: 24804884]

19. Mikolasek M, Witt CM, Barth J. Adherence to a mindfulness and relaxation self-care app for cancer patients: mixed-methods feasibility study. JMIR Mhealth Uhealth 2018 Dec 6;6(12):e11271 [FREE Full text] [doi: 10.2196/11271] [Medline: $\underline{30522990]}$

20. Rosen K, Potter J. Participant engagement in a commercially available app-based mindfulness training intervention delivered to women diagnosed with breast cancer. iproc 2018 Sep;4(2):e11907. [doi: 10.2196/11907]

21. Rosen KD, Paniagua SM, Kazanis W, Jones S, Potter JS. Quality of life among women diagnosed with breast Cancer: A randomized waitlist controlled trial of commercially available mobile app-delivered mindfulness training. Psychooncology 2018 Aug;27(8):2023-2030. [doi: 10.1002/pon.4764] [Medline: 29766596]

22. Huberty J, Eckert R, Larkey L, Joeman L, Mesa R. Experiences of using a consumer-based mobile meditation app to improve fatigue in myeloproliferative patients: qualitative study. JMIR Cancer 2019 Jul 22;5(2):e14292 [FREE Full text] [doi: 10.2196/14292] [Medline: 31333197$]$

23. Grewal N, Arya R, Malik R, Hasan Y, Golden D, McCall A. Implementing a tablet-guided mindfulness meditation intervention to reduce anxiety in women receiving radiotherapy for breast cancer. Int J Radiat Oncol Biol Phys 2019 Sep;105(1):E588. [doi: 10.1016/j.ijrobp.2019.06.1181]

24. Noone C, Hogan MJ. A randomised active-controlled trial to examine the effects of an online mindfulness intervention on executive control, critical thinking and key thinking dispositions in a university student sample. BMC Psychol 2018 Apr 5;6(1):13 [FREE Full text] [doi: 10.1186/s40359-018-0226-3] [Medline: 29622047]

25. Puddicombe A. The Headspace Guide to Meditation and Mindfulness: How Mindfulness Can Change Your Life in Ten Minutes a Day. New York, NY: St. Martin's Griffin; 2012. 
26. Bruce NG, Bruce NG, Shapiro SL, Constantino MJ, Manber R. Psychotherapist mindfulness and the psychotherapy process. Psychotherapy (Chic) 2010 Mar;47(1):83-97. [doi: 10.1037/a0018842] [Medline: 22402003]

27. Donovan E, Martin SR, Seidman LC, Zeltzer LK, Cousineau TM, Payne LA, et al. A mobile-based mindfulness and social support program for adolescents and young adults with sarcoma: development and pilot testing. JMIR Mhealth Uhealth 2019 Mar 18;7(3):e10921 [FREE Full text] [doi: 10.2196/10921] [Medline: 30882352]

28. Sun Y, Zhang Y, Gwizdka J, Trace CB. A mobile-based mindfulness and social support program for adolescents and young adults with sarcoma: development and pilot testing. J Med Internet Res 2019 May 2;21(5):e12522 [FREE Full text] [doi: 10.2196/12522] [Medline: 31045507]

29. Borah P, Xiao X. The importance of 'likes': the interplay of message framing, source, and social endorsement on credibility perceptions of health information on Facebook. J Health Commun 2018;23(4):399-411. [doi: 10.1080/10810730.2018.1455770] [Medline: 29601271]

30. Donovan E, Zeltzer L, Federman N, Cousineau T, Payne L, Martin S, et al. A patient-centered approach to tailoring a mobile-based mindfulness and social support intervention for adolescents and young adults with cancer. iproc 2017 Sep 22;3(1):e25 [FREE Full text] [doi: 10.2196/iproc.8408]

31. Mobio Interactive. Team URL: https://www.midigitaltherapeutics.com/team.html [accessed 2019-11-21]

32. Mobio Interactive. R\&D URL: https://www.midigitaltherapeutics.com/rd.html [accessed 2019-11-21]

33. Walsh KM, Saab BJ, Farb NA. Effects of a mindfulness meditation app on subjective well-being: active randomized controlled trial and experience sampling study. JMIR Ment Health 2019 Jan 8;6(1):e10844 [FREE Full text] [doi: 10.2196/10844] [Medline: 30622094]

34. Costa MA, de Oliveira GS, Tatton-Ramos T, Manfro GG, Salum GA. Anxiety and stress-related disorders and mindfulness-based interventions: a systematic review and multilevel meta-analysis and meta-regression of multiple outcomes. Mindfulness 2019;10(6):996-1005. [doi: 10.1007/s12671-018-1058-1]

35. Faul F, Erdfelder E, Lang A, Buchner A. G*Power 3: a flexible statistical power analysis program for the social, behavioral, and biomedical sciences. Behav Res Methods 2007 May;39(2):175-191. [doi: 10.3758/bf03193146] [Medline: 17695343]

36. Kubo A, Kurtovich E, McGinnis M, Aghaee S, Altschuler A, Quesenberry C, et al. A randomized controlled trial of mHealth mindfulness intervention for cancer patients and informal cancer caregivers: a feasibility study within an integrated health care delivery system. Integr Cancer Ther 2019;18:1534735419850634 [FREE Full text] [doi: 10.1177/1534735419850634] [Medline: 31092044]

37. Harris PA, Taylor R, Thielke R, Payne J, Gonzalez N, Conde JG. Research electronic data capture (REDCap)--a metadata-driven methodology and workflow process for providing translational research informatics support. J Biomed Inform 2009 Apr;42(2):377-381 [FREE Full text] [doi: 10.1016/j.jbi.2008.08.010] [Medline: 18929686]

38. Mobio Interactive Inc. URL: https://www.midigitaltherapeutics.com/ [accessed 2019-07-01]

39. Twilio - Communication APIs for SMS, Voice, Video and Authentication. URL: https://www.twilio.com [accessed 2019-11-21]

40. Jason D, Audrey C, Brendan C, Robert A, Farnoud K, Xiao Yu W, et al. Photoplethysmographic Imaging via Spectrally Demultiplexed Erythema Fluctuation Analysis for Remote Heart Rate Monitoring. In: Proceedings of Multimodal Biomedical Imaging XI, Volume 9701. 2016 Presented at: SPIE'16; February 13-14, 2016; San Francisco URL: https://doi.org/10.1117/ 12.2213532 [doi: $10.1117 / 12.2213532]$

41. Al-Jebrni A, Chwyl B, Wang X, Wong A, Saab BJ. AI-enabled remote and objective quantification of stress at scale. Biomed Signal Process Control 2020 (forthcoming)(forthcoming). [doi: 10.1016/j.bspc.2020.101929]

42. Carlson LE. Clinical Trials. 2019. The SEAMLESS Study: Smartphone App-based Mindfulness for Cancer Survivors URL: https://clinicaltrials.gov/ct2/show/NCT03557762 [accessed 2018-09-29]

43. Eysenbach G, CONSORT-EHEALTH Group. CONSORT-EHEALTH: improving and standardizing evaluation reports of Web-based and mobile health interventions. J Med Internet Res 2011 Dec 31;13(4):e126 [FREE Full text] [doi: 10.2196/jmir.1923] [Medline: 22209829]

44. Boutron I, Altman DG, Moher D, Schulz KF, Ravaud P, CONSORT NPT Group. CONSORT statement for randomized trials of nonpharmacologic treatments: a 2017 update and a CONSORT extension for nonpharmacologic trial abstracts. Ann Intern Med 2017 Jul 4;167(1):40-47. [doi: 10.7326/M17-0046] [Medline: 28630973]

45. Broderick J, DeWitt EM, Rothrock N, Crane P, Forrest CB. Advances in patient-reported outcomes: The NIH PROMIS(®) Measures. EGEMS (Wash DC) 2013;1(1):1015 [FREE Full text] [doi: 10.13063/2327-9214.1015] [Medline: 25848562]

46. Callahan CM, Unverzagt FW, Hui SL, Perkins AJ, Hendrie HC. Six-item screener to identify cognitive impairment among potential subjects for clinical research. Med Care 2002 Sep;40(9):771-781. [doi: 10.1097/00005650-200209000-00007] [Medline: 12218768 ]

47. Carlson LE, Thomas BC. Development of the Calgary Symptoms of Stress Inventory (C-SOSI). Int J Behav Med 2007;14(4):249-256. [doi: 10.1007/bf03003000] [Medline: 18001241]

48. Simard S, Savard J. Fear of Cancer Recurrence Inventory: development and initial validation of a multidimensional measure of fear of cancer recurrence. Support Care Cancer 2009 Mar;17(3):241-251. [doi: 10.1007/s00520-008-0444-y] [Medline: 18414902] 
49. Carlson LE, Brown KW. Validation of the Mindful Attention Awareness Scale in a cancer population. J Psychosom Res 2005 Jan;58(1):29-33. [doi: 10.1016/j.jpsychores.2004.04.366] [Medline: 15771867]

50. Trapnell PD, Campbell JD. Private self-consciousness and the five-factor model of personality: distinguishing rumination from reflection. J Pers Soc Psychol 1999 Feb;76(2):284-304. [doi: 10.1037//0022-3514.76.2.284] [Medline: 10074710]

51. Hayes SC, Wilson KG, Gifford EV, Follette VM, Strosahl K. Experimental avoidance and behavioral disorders: a functional dimensional approach to diagnosis and treatment. J Consult Clin Psychol 1996 Dec;64(6):1152-1168. [doi: 10.1037//0022-006x.64.6.1152] [Medline: 8991302]

52. Cella D, Riley W, Stone A, Rothrock N, Reeve B, Yount S, PROMIS Cooperative Group. The Patient-Reported Outcomes Measurement Information System (PROMIS) developed and tested its first wave of adult self-reported health outcome item banks: 2005-2008. J Clin Epidemiol 2010 Nov;63(11):1179-1194 [FREE Full text] [doi: 10.1016/j.jclinepi.2010.04.011] [Medline: 20685078]

53. Schalet BD, Pilkonis PA, Yu L, Dodds N, Johnston KL, Yount S, et al. Clinical validity of PROMIS Depression, Anxiety, and Anger across diverse clinical samples. J Clin Epidemiol 2016 May;73:119-127 [FREE Full text] [doi: 10.1016/i.jclinepi.2015.08.036] [Medline: 26931289]

54. Cella D, Lai J, Jensen SE, Christodoulou C, Junghaenel DU, Reeve BB, et al. PROMIS fatigue item bank had clinical validity across diverse chronic conditions. J Clin Epidemiol 2016 May;73:128-134 [FREE Full text] [doi: 10.1016/j.jclinepi.2015.08.037] [Medline: 26939927]

55. Jensen RE, Potosky AL, Reeve BB, Hahn E, Cella D, Fries J, et al. Validation of the PROMIS physical function measures in a diverse US population-based cohort of cancer patients. Qual Life Res 2015 Oct;24(10):2333-2344 [FREE Full text] [doi: 10.1007/s11136-015-0992-9] [Medline: 25935353]

56. Lengacher CA, Shelton MM, Reich RR, Barta MK, Johnson-Mallard V, Moscoso MS, et al. Mindfulness based stress reduction (MBSR(BC)) in breast cancer: evaluating fear of recurrence (FOR) as a mediator of psychological and physical symptoms in a randomized control trial (RCT). J Behav Med 2014 Apr;37(2):185-195 [FREE Full text] [doi: 10.1007/s10865-012-9473-6] [Medline: 23184061]

\author{
Abbreviations \\ ACR: Alberta Cancer Registry \\ CONSORT: Consolidated Standards of Reporting Trials \\ C-SOSI: Calgary Symptoms of Stress Inventory \\ ITT: intent to treat \\ LMM: linear mixed model \\ MBCR: Mindfulness-Based Cancer Recovery \\ MBCS: Mindfulness-Based Cancer Survivorship \\ MBI: mindfulness-based interventions \\ MI: Mobio Interactive Inc \\ PROMIS: Patient-Reported Outcome Information System \\ REDCap: Research Electronic Data Capture
}

Edited by G Eysenbach; submitted 19.08.19; peer-reviewed by E Kent, C Roos; comments to author 15.10.19; revised version received
27.11.19; accepted 04.02.20; published 11.05.20
Please cite as:
Subnis UB, Farb NAS, Piedalue KAL, Speca M, Lupichuk S, Tang PA, Faris P, Thoburn M, Saab BJ, Carlson LE
A Smartphone App-Based Mindfulness Intervention for Cancer Survivors: Protocol for a Randomized Controlled Trial
JMIR Res Protoc 2020;9(5):e15178
URL: 1 https://www.researchprotocols.org/2020/5/e15178
doi: $\underline{10.2196 / 15178}$
PMID: $\underline{32390591}$

(CUtkarsh B Subnis, Norman AS Farb, Katherine-Ann Laura Piedalue, Michael Speca, Sasha Lupichuk, Patricia A Tang, Peter Faris, Mark Thoburn, Bechara J Saab, Linda E Carlson. Originally published in JMIR Research Protocols (http://www.researchprotocols.org), 11.05.2020. This is an open-access article distributed under the terms of the Creative Commons Attribution License (https://creativecommons.org/licenses/by/4.0/), which permits unrestricted use, distribution, and reproduction in any medium, provided the original work, first published in JMIR Research Protocols, is properly cited. The complete bibliographic information, a link to the original publication on http://www.researchprotocols.org, as well as this copyright and license information must be included. 